\title{
Voluntariado Corporativo y Responsabilidad de la Empresa: Aproximación a la Gestión de los Recursos Humanos
}

\author{
José Paulo Cosenza ${ }^{1 *}$, \\ Faculdade de Administração e Ciências Contábeis - Universidade Federal Fluminense, Niterói - RJ, Brasil \\ María Isabel Saz Gil', \\ Facultad de Ciencias Sociales y Humanas - Universidad de Zaragoza, Zaragoza - Espanha \\ Ana Isabel Zardoya Alegria, \\ Facultad de Economía y Empresa - Universidad de Zaragoza, Zaragoza - Espanha
}

Recebido em 20 de Junho de 2016. Aceito em 12 de Agosto de 2016

\section{RESUMO}

O crescimento e a consolidação do voluntariado corporativo passam, necessariamente, pela melhoria da qualidade e da gestão do trabalho voluntário, conforme seus aspectos motivadores, já que contempla um recurso humano com características especiais. Neste trabalho pretendemos discutir a questão da relação da motivação com a gestão do voluntariado corporativo, tendo em vista que gera impactos na conduta de diferentes grupos de interesse. Particularmente, o trabalho tem o objetivo de realizar uma aproximação, sob uma perspectiva teórico-analítica, do ciclo de voluntario na empresa com a gestão dos recursos humanos, analisando seus efeitos e sinergias para o voluntariado corporativo, em termos de motivação e avaliação dos resultados.

Palavras-chave: Voluntariado empresarial; Responsabilidade social corporativa; competências; Ciclo voluntário. ABSTRACT

The growth and consolidation of corporate volunteering necessarily involve improving the quality and management of voluntary work, in their motivational aspects, since it includes human resources with special characteristics. In this paper we intend to discuss the relationship between motivation and corporate volunteering management, in order to generate impacts on the behavior of different stakeholders. This study aims specifically to approach, from a theoretical and analytical perspective, the business volunteering cycle and human-resources management, analyzing their effects and synergies for corporate volunteering, in terms of motivation and results evaluation.

Keywords: Corporate Volunteering, Corporate Social Responsibility, Competitions, Volunteering Cycle.

\section{RESUMEN}

El crecimiento y la consolidación del voluntariado corporativo pasan, necesariamente, por mejorar la calidad y la gestión del trabajo voluntario, según sus aspectos motivadores, ya que representa un recurso humano con especiales características. En este trabajo pretendemos discutir la cuestión de la relación de la motivación con la gestión del voluntariado corporativo, teniendo en cuenta que genera impactos en la conducta de diferentes grupos de interés. En concreto el trabajo tiene el objetivo de realizar una aproximación, desde una perspectiva teórico-analítica, al ciclo del voluntario en la empresa y a la gestión de los recursos humanos, analizando sus efectos y sinergias para el voluntariado corporativo.

Palabras Clave: Voluntariado Corporativo; Responsabilidad Social Corporativa; Competencias; Ciclo del Voluntariado.

1. Professor Titular da Faculdade de Administração e Ciências Contábeis - Universidade Federal Fluminense

2. Professora Ayudante da Facultad de Ciencias Sociales y Humanas- Universidad de Zaragoza

3. Professora Titular da Facultad de Economía y Empresa - Universidad de Zaragoza

* Autor para correspondência. José Paulo Cosenza

e-mail: jpcosenza@id.uff.br 


\section{Introducción}

Históricamente el trabajo de voluntariado estuvo siempre relacionado preponderantemente con las acciones de caridad, la gran mayoría asociada a instituciones religiosas. Sin embargo, en los últimos años, el voluntariado está asumiendo nuevos diseños en su forma de trabajo, hecho que está exigiendo mayor atención e incentivo de los gobiernos, empresas, de las propias Organizaciones no Lucrativas (en adelante ONL) y, también, de las personas individualmente. Según Souza (2008), el trabajo voluntario que antes estaba ligado mayoritariamente a la caridad cristiana, actualmente se presenta en gran parte laicizado y vinculado a iniciativas empresariales y a la manifestación de la acción ciudadana.

En los años setenta en Estados Unidos surgió un tipo particular de trabajo voluntario asociado a la Responsabilidad Social de la Empresa (en adelante RSE). En concreto, esa modalidad de trabajo voluntario se constituyó en acciones sociales desarrolladas por fuerza de una iniciativa empresarial bajo la cual, en general, se movilizaba el trabajo voluntario de los empleados de dichas corporaciones o incluso de la comunidad en general, con o sin aporte financiero directo de la empresa patrocinadora.

Desde entonces una de las grandes vertientes del trabajo voluntario ha sido conducida por las empresas que motivadas por el reto de la Responsabilidad Social Corporativa (en adelante RSC) y estimuladas no solamente por la legislación patria, pero también por diversos movimientos globales, asumen la responsabilidad de incrementar esfuerzos por un mundo mejor y de desarrollar en sus funcionarios la satisfacción por participar de una organización que se les apoya y se les estimula en la implantación de proyectos y programas de trabajo voluntario (LIMA y BARELI, 2011). De ahí que las empresas invierten cada vez más en acciones sociales, no solo por esa creciente concienciación o por fuerza de ley, sino también para ser diferenciadas y competitivas en sus mercados, con el compromiso mayor de sus trabajadores.

En este contexto emerge el Voluntariado Corporativo (en adelante VC) como una nueva tendencia en el marco de las políticas de RSC que implementan las empresas, en particular las grandes empresas. Sin embargo, aunque de gran importancia y significado para concretar la RSE, entre las particularidades que definen los programas de VC está la de que generan impacto en diferentes grupos de interés, destacando la participación de la empresa y de sus empleados. Por ello, requiere de un alto nivel de planificación y coordinación entre todas las partes implicadas.

\section{Metodología}

El objetivo de este trabajo es contribuir a la mejora del conocimiento y de la gestión del VC incidiendo en los efectos positivos que para los recursos humanos de las empresas puede tener la implementación de programas de VC, identificando las fases y los factores a tener en cuenta para facilitar el proceso de control de gestión y garantizar el impacto deseado en la conducta de los recursos humanos. En esta línea, el desarrollo de este estudio se justifica por tres cuestiones fundamentales. Primero, por aportar conocimiento respecto a un tema (el VC) que es cada vez más una parte de la RSE y que implica necesariamente el establecimiento de mecanismos de control de gestión, en especial relacionándolo con la política de recursos humanos. En segundo lugar, por presentar una propuesta de colaboración estable entre la empresa y la entidad de voluntariado. Y, en tercer lugar, por permitir fomentar iniciativas dirigidas a mejorar, en general, la capacitación del personal directivo, gestor y profesional que actúan en el VC.

Además, 2015 es un año crucial, en todo el mundo, para el desarrollo de las actividades de VC porque se pone en marcha el "Impact 2030", que es una iniciativa, liderada por el sector empresarial y el Pacto Global de las Naciones Unidas, para poner en valor el VC. Según señalado en el Observatorio de Voluntariado Corporativo, se trata de convertir el VC en la gran herramienta de servicio del sector privado a toda la sociedad, para alcanzar los nuevos Objetivos de Desarrollo Sostenible (SDO), presentados en La Organización de las Naciones Unidas (ONU) para sustituir a los Objetivos de Desarrollo del Milenio (MDO). 
Nuestra contribución con el presente estudio radica en realizar una aproximación del VC con la gestión de los recursos humanos, analizando sus efectos y sinergias dentro de la estrategia general de Responsabilidad Social (en adelante RS) de las empresas. En esta línea, nuestro problema de investigación es discutido dentro del ámbito de la Contabilidad de Gestión, según la perspectiva de la RSC. Para eso tomamos como referencia el marco teórico sobre el VC intentando incorporarlo como un elemento clave en la estrategia de responsabilidad corporativa de la empresa y también como una nueva actividad en la gestión empresarial que genera valor para las organizaciones, para la sociedad civil y para los recursos humanos.

En consecuencia, nuestro análisis está desarrollado siguiendo un diseño metodológico propio de una investigación de característica exploratorio-descriptiva (RICHARDSON, 1999), donde a partir del planteamiento de un ensayo conceptual sobre el tema investigado, buscamos poner de manifiesto los puntos más relevantes asociados a la aplicación en la práctica en la empresa del VC. A partir de nuestra interpretación analítica del marco conceptual y la exposición descriptiva revisamos la bibliografía teórica pertinente sobre el tema y aportamos nueva mirada al tema analizado, emitiendo juicios sobre el carácter del VC y el papel de los recursos humanos desde una perspectiva en línea con la estrategia corporativa y la RSC.

\section{Voluntariado corporativo y motivación en el trabajo}

Definido como el conjunto de actividades promovidas y apoyadas por una empresa que tiene como finalidad la involucración y participación libre de sus empleados a través de la dedicación de su tiempo, capacidades y talento a causas, proyectos y organizaciones sin ánimo de lucro, las actividades del VC se desarrollan en el marco de la empresa que no se limita simplemente a ser un actor que influye para que los empleados realicen este tipo de actividades, sino que los apoya destinando recursos económicos, en especie y tiempo (CES y ADECCO, 2011, p. 18).

Así, el VC consiste en el ejercicio y participación de una empresa en actividades de voluntariado realizadas por sus empleados. Esas actividades surgen como una respuesta a las demandas externas de colaboradores y de la sociedad civil (Houghton et al., 2009) e internas en la motivación del capital humano (HARRIS, 2000) frente al compromiso responsable de la empresa e implican para su gestión diversas áreas de la estructura organizativa de la empresa.

Sim embargo, es complicado presentar un concepto concreto y único de VC (GALLARDO y SANCHEZ, 2014, p. 7), por tratarse de un área de estudio aún carente de sustentación teórica propia (TSCHIRHART, 2005). Aunque pueda parecer que el VC tenga efectos positivos en la organización, hay pocas evidencias empíricas que lo demuestren (GALLARDO et al, 2010, p. 61). Así, destacamos la importancia del VC estar articulado en la estrategia de la empresa, en particular a la RS, a la vez que subraya la importancia del vínculo entre la empresa, los recursos humanos, los stakeholders y la sociedad civil.

De ahí que, actualmente, el VC está siendo utilizado como un recurso estratégico de las empresas buscando fortalecer la imagen empresarial en el ámbito de la RSC ya que constituye una expresión importante de la puesta en práctica de acciones de RS. Sin embargo, también se le puede usar como mecanismo conductor para capacitar competencias en las personas, y motivarlas en su trabajo, planteamientos estos que serán analizados en este trabajo.

Esto es lo que pretendemos con la elaboración de este estudio porque ese concepto hace dos décadas ni se planteaba en el ámbito corporativo ya que a simple vista parece una contradicción intentar encajar los términos "empresa" y "voluntariado". Sin embargo, dichos términos, lejos de ser antagónicos, son una combinación poderosa que bien gestionada reporta un evidente beneficio social a la comunidad a la vez que contribuye a que aquella consiga sus objetivos de negocio de manera más rentable (CES y ADECCO, 2011, p. 8). En ese sentido, las actividades de VC están experimentando una evolución estratégica motivando el desarrollo de nuevas iniciativas (por ejemplo, proyectos de voluntariado profesional, vacaciones solidarias o excedencias laborales), en las que la búsqueda de nuevas oportunidades para potenciar el desarrollo de competencias y fomentar nuevas habilidades comienza a revelarse como elemento clave. 
Según Kotler y Lee (2005), el VC se caracteriza como la estrategia que permite a la empresa motivar a sus empleados a donar talentos, ideas y esfuerzos físicos en favor de las causas de la comunidad local. Aunque no van a recibir ningún tipo de recompensa material o financiera como pago por las horas dedicadas al trabajo voluntario, sus esfuerzos son un importante medio por el cual se les puede atribuir responsabilidades, haciéndoles activamente conscientes de que ejercen un relevante papel en el proceso de cambio de la realidad social (VERAS, 2012).

Desde el punto de vista de la conducta, el control de la evasión de los empleados es la cuestión crítica para el éxito del VC (SILVA y FEITOSA, 2002; TEODÓSIO, 1999), siendo la falta de claridad en cuanto a las motivaciones y expectativas que llevaron a la persona a ingresar en el trabajo voluntario una de las posibles causas de dicho abandono (COMUNIDADE SOLIDÁRIA, 1997). Existe una amplia literatura empírica, parte de ella ya clásica (MASLOW, 1954; HERZBERG, 1966; SIMON, 1965; MCCLELLAND, 1965; ALDERFER, 1972; LOCCKE, 1968; y VROOM, 1964; de entre otros), que han estudiado la cuestión de la motivación en las organizaciones. Sin embargo, para Cardona, Lawrence y Espejo (2003), aunque varias líneas teóricas hayan intentado investigar el efecto de diferentes incentivos sobre la motivación, pocas han demostrado el real efecto de la motivación en el trabajo desde el prisma de los resultados de las acciones individuales.

Según Lathan y Pinder (2005), la motivación para el trabajo es un conjunto de fuerzas que impulsan un individuo a empezar un comportamiento asociado al trabajo y a determinar su forma, dirección, intensidad y duración. Pero como la motivación es un proceso psicológico complejo y resultante de una interacción entre el individuo y el entorno que se le rodea (LATHAM y PINDER, 2005), son muchos los factores que pueden tener influencia sobre las motivaciones (PITTMAN y HELLER, 1987).

Para Cardona, Lawrence y Espejo (2003), una gran parte de las teorías sobre la motivación están centradas en analizar la motivación extrínseca y su influencia en el comportamiento de las personas. Pero hay evidencias crecientes de que otros tipos de incentivos que no son recompensas contingentes pueden también motivar a las personas a trabajar, por ejemplo el aprender, el deseo de pertenecer, o la voluntad para contribuir (BAUMEISTER y LEARY, 1995).

El VC es un conjunto de acciones que la empresa desarrolla para motivar y apoyar el compromiso de sus trabajadores en actividades voluntarias en la comunidad (GOLDBERG, 2001). Sin embargo, no debemos olvidar que la gestión del trabajo voluntariado tiene como base el modelo de gestión empresarial (ALVES, 2010). Además, no se puede también olvidar que los voluntarios precisan obtener más satisfacción en sus realizaciones justamente porque no reciben ningún tipo de remuneración material o financiera (CAMARGO et al, apud COSTA, 2004). Quizás es por eso que Garay y Mazzilli (2003) identifican al VC como una forma de entrenamiento, capaz de ofrecer la oportunidad para el ejercicio del liderazgo y de la responsabilidad, mientras aumenta el nivel de satisfacción e identidad de los empleados con la empresa.

Para Amabile y Cramer (2012) la motivación es una combinación de la elección de una persona para realizar una tarea, su deseo de dedicar su esfuerzo a realizarla y su impulso para persistir en ese esfuerzo. Aunque las posibles fuentes de motivación en el entorno laboral sean las más variadas, los mencionados autores señalan tres motivaciones como las más importantes:

i) Extrínseca: hacer algo con el fin de lograr otra cosa;

ii) Intrínseca: surge del amor al propio trabajo y del querer hacerlo bien porque es interesante, satisfactorio y fascinante o personalmente estimulante; y iii) Relacional: también conocida como altruista, surge de la necesidad de conectar con y ayudar a los demás.

Es decir, los autores añaden a los dos tipos de motivación ya clásicos (extrínseca e intrínseca) una tercera, la relacional en donde tiene cabida el VC.

$\mathrm{Al}$ analizar los aspectos que son motivadores del desarrollo del trabajo voluntario, Corullón (1997) señala haber descubierto, entre otros, dos componentes fundamentales: 
(a) Personal: relacionado a la donación de tiempo y esfuerzo como respuesta a una inquietud interior que es conducida a la práctica; $y$

(b) Social: asociado a la toma de conciencia de los problemas al enfrentarse con la realidad, o que lleva a la lucha por un ideal o a comprometerse por una causa.

Teodósio (2001) señala que los voluntarios son motivados por un espacio de convivencia social más saludable, huyendo de la competitividad y del estrés que caracterizan el trabajo en el área privada.

Según Geber (1991), las personas participan del trabajo voluntario por tres razones principales:

(i) Realización: personas a quién apetece dar algo a la sociedad;

(ii) Afiliación: voluntarios que tienen el deseo de conocer otras personas; y

(iii) Poder: utilizando el voluntariado como un camino más sencillo para alcanzar posiciones de liderazgo más aún que esperar una promoción en el trabajo.

Además de esto, Azevedo (2007), detectó que motivaciones como altruismo y solidaridad, ayudados por una dedicación espontanea del voluntariado, tienden a ser mezclados cada vez más con intereses privados de perfeccionamiento de currículo y vivencia profesional.

A este respecto, Azevedo (2007), contrastó el trabajo voluntario desde cinco categorías de motivación:

(1) Asistencial: perspectiva de ayudar el otro que es carente de afecto, cosas materiales y conocimiento;

(2) Humanitaria: contribuir con el otro, visto como semejante y próximo, pudiendo incluirse el crecimiento espiritual;

(3) Política: búsqueda del ejercicio de la ciudadanía con acción emancipadora;

(4) Profesional: experimentar conocimientos adquiridos en la universidad, aplicarlos para obtener empleo en ONG; y

(5) Personal: vinculada al tratamiento terapéutico y a la búsqueda de relacionamiento interpersonal y de retorno emocional.

Siguiendo esta línea, excluyéndose las cuestiones profesionales, de afiliación y religiosas, se puede plantear una visión más endémica de las motivaciones del trabajo voluntario donde se puede identificar las motivaciones para el trabajo voluntario alrededor de las siguientes cuatro perspectivas:

$1^{\text {a) }}$ Asistencial: pensar en el próximo y ayudarle sin esperar nada en cambio, en otras palabras, se importar con la condición de vida de quien es menos favorecido y hacer un poquito para él, donando su tiempo y conocimiento para contribuir con el bien estar de las personas;

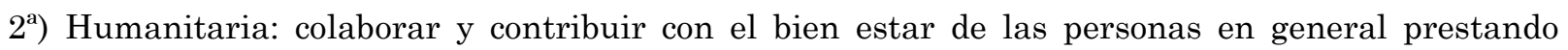
servicios, dedicando tiempo, talento y habilidad;

$\left.3^{a}\right)$ Ideológica: acción política o de ciudadanía buscando cambiar o construir un mundo mejor o un país con menos desigualdades y más amor y justicia social; y

$4^{\mathrm{a}}$ ) Realización: sentir placer y gratificado personalmente por hacer lo que haz, en otras palabras, tener algo a ofrecer.

\section{Voluntariado corporativo en la estrategia competitiva}

El VC surge en los años setenta en Estados Unidos. En Europa el reconocimiento del año 2011 como Año Europeo de las Actividades de Voluntariado que Fomenten una Ciudadanía Activa sirvió para impulsar el voluntariado, y concretamente para promover iniciativas de creación de redes que estimulen las sinergias entre organizaciones de voluntariado y otros sectores especialmente las empresas. En España, su desarrollo es incipiente y está asociado principalmente a grandes empresas, que en algunos casos empiezan con algunos sectores de sus recursos humanos; como los jubilados y prejubilados y 
posteriormente van avanzando en otros sectores. También se extienden a otras entidades que actúan en otros países y que necesitan voluntariado. En el caso brasileño, el inicio del VC se enmarca en el año 1998 con la promulgación de la Ley $\mathrm{n}^{\circ}$ 9.608, de 18 de febrero). Esa Ley del Voluntariado ganó destaque a partir del año 1998 con el Programa de Voluntarios organizado por el Consejo de Comunidad Solidaria que buscaba promover encuentros, fomentar iniciativas y propiciar más visibilidad a las prácticas de voluntariado existentes en Brasil (ROSENFELD, MUTERLE y GASPAR, 2013).

El VC vincula temas de gran importancia, tanto en la empresa como en la ONL. Por un lado, está vinculado, en la empresa, a las estrategias de RS y de recursos humanos y a la inversión socialmente responsable y, por otro lado, contribuye al diálogo entre sector empresarial y tercer sector. En el VC las empresas ofrecen a sus trabajadores la opción de participar en una ONL como voluntariado, desarrollando tareas que ellos pueden desempeñar. Así en el VC hay tres actores implicados: i) los empleados; ii) la empresa; y iii) las ONL. Los empleados trabajan sintiéndose más motivados e incentivados. La empresa gana puntos ante su entorno y todo ello redunda en un aumento de su beneficio tanto por la mejora de sus recursos humanos como por su imagen y reputación (GALLARDO et al, 2010). Las ONL se benefician directamente por el aumento de su voluntariado, y también porque reciben voluntariado con habilidades o destrezas, como el uso de las nuevas tecnologías, sistemas de comunicación y sistemas de contabilidad (ALLEN, 2003).

En el contexto actual, las empresas deben crear valor no solo a nivel de obtención de beneficios, sino también a nivel social. La RS es uno de los pilares sobre los que se asienta el concepto de VC. Según la RS, además de los resultados económico-financieros, la empresa debe trabajar para satisfacer un triple objetivo: social, económico y medioambiental "triple bottom line" (MCDONOUGH y BRAUNGART, 2002).

Otro de los pilares del VC es la teoría stakeholder, según la cual la realización de cualquier actividad empresarial ha de ir encaminada a la satisfacción de los intereses de las distintas partes implicadas. Hemos pasado de un entorno en el que apenas había otro objetivo que la remuneración de los accionistas a través de los dividendos y ellos eran prácticamente los únicos a los que iba dirigida la información financiera de la empresa, a otro en el cual hay variados agentes implicados e interesados en su actividad.

Por otro lado, hay que señalar que en concreto, la motivación es uno de los temas de mayor importancia en el ámbito empresarial debido, principalmente, al amplio margen de maniobra que se proporciona a los gerentes en la toma de decisiones y en la selección de técnicas, métodos y criterios que muchas veces pueden permitir a estos ejecutivos satisfacer más a sus intereses propios que a los de la entidad que representan. Por esta razón, el estudio de la compensación ejecutiva ha despertado el interés de una gran cantidad de investigadores académicos, en particular de los teóricos del tema de la agencia. Aunque dichos trabajos han intentado comprender la relación entre el desempeño administrativo y el esquema de incentivos, Prendergast (1999) entiende que han sido desarrollados pocos estudios empíricos tratando los efectos de los incentivos para la compensación de los trabajadores.

Sin embargo, en la actualidad la teoría de los Stakeholders y la RSC son las dos teorías que determinan el funcionamiento óptimo de las organizaciones (GALLARDO et al, 2010). La primera persigue la satisfacción de las necesidades de todos los agentes o grupos de interés involucrados en el desarrollo de la gestión empresarial. La segunda se puede definir como "el reconocimiento e integración en sus operaciones por parte de las empresas, de las preocupaciones sociales y medioambientales, dando lugar a prácticas empresariales que satisfagan dichas preocupaciones y configuren sus relaciones con sus interlocutores" (DE LA CUESTA y VALOR, 2003). Estas dos teorías están ligadas y son la base del VC. El VC tiene que implantarse a nivel estratégico, porque hace que la empresa sea socialmente responsable y también porque puede conseguir una verdadera motivación en el área de recursos humanos, muy difícil de alcanzar en muchas ocasiones por los métodos tradicionales de control de gestión.

Así, según el estudio del Club de Excelencia en Sostenibilidad con la Fundación Adecco (2011) sobre el estado del VC en España, la gran mayoría de las ventajas y beneficios que aporta el VC a las empresas están relacionadas con el área de los recursos humanos. 
Entre las muchas ventajas que se citan destacamos, a modo de resumen, las siguientes:

- Mejora la relación de la dirección con los empleados.

- Se crean nuevas relaciones entre los empleados de los diferentes departamentos.

- Ayuda a que los empleados desarrollen habilidades de liderazgo, trabajo en equipo y creatividad.

- Genera un sentimiento de orgullo de pertenencia a la empresa.

- Genera una visión global de la empresa, que va más allá de las limitaciones de un puesto o un departamento.

- Eleva la moral de los empleados, generándoles un sentimiento de satisfacción.

En esta misma línea Sanz et al (2012) explican que además de los beneficios que la empresa obtiene con esta herramienta para la comunidad en general, las empresas han reconocido también en el VC a una buena política de recursos humanos, con unas repercusiones importantes en cuestiones como el fortalecimiento de valores éticos entre los empleados, la promoción de valores como el trabajo en equipo, y la mejora del clima laboral, del sentido de pertenencia a la empresa o incluso de su imagen, tanto interna como externa.

No queremos terminar este apartado sin resaltar de nuevo la vinculación que debe tener el VC con el ámbito estratégico de la empresa, ya que éste puede utilizarse para mejorar las competencias en desarrollo empresarial y fomentar la capacidad de innovar, además de la mejora en la capacidad de liderazgo en el ámbito de los recursos humanos. En el caso del VC para el desarrollo puede servir para dirigir o afianzar la estrategia de la empresa hacia mercados emergentes, gracias a la inmersión en nuevas culturas y contextos, los empleados pueden comprender mejor sus hábitos y sus comportamientos. Esto puede apoyar la creación de estrategias de comercialización más pertinentes, aunque con las limitaciones de la complicada adaptación de los sistemas de gestión a estos mercados y del coste que supone.

\section{Aproximación a la gestión del voluntariado corporativo}

El VC lleva aparejado cierta complejidad para la empresa, como señalan (GALLARDO et al, 2010) en el VC se produce un intercambio en el que intervienen tres actores diferentes (empresa, empleados y ONL), y cada uno con sus objetivos y expectativas, por ello el VC ha de ser capaz de constituirse como un escenario en el que todos ganen (PELOZA y HASSAY, 2006). De acuerdo con Allen (2012), el VC cuenta con unas características específicas que tienen que observarse para desarrollar un programa de VC. Siguiendo a Allen (2012), un programa de VC ha de estar alineado con las competencias de la empresa; estar basado en habilidades profesionales y en la experiencia laboral de los empleados de la empresa; centrarse en el problema a resolver y en los beneficiarios; tratar de desarrollar el liderazgo y las habilidades de los empleados que participan; seguir un proceso riguroso y serio en el desarrollo del programa con elevados estándares.

En un programa de VC la empresa establece alianzas y trabaja en estrecha colaboración con la ONL. Las empresas buscan de forma deliberada aprender sobre y de la experiencia de sus empleados voluntarios. Por lo que un programa de VC se debe estructurar de forma similar al proceso que sigue cualquier organización al establecer su control de gestión según el ciclo básico de retroalimentación (Figura 1), en el que se identifican las etapas de planificación, implementación, evaluación y actuación. En este sentido, la Fundación CODESPA y la consultora internacional The Boston Consulting Group han formalizado un proceso de implementación de programas de VC (FUNDACIÓN CODESPA, 2012). 


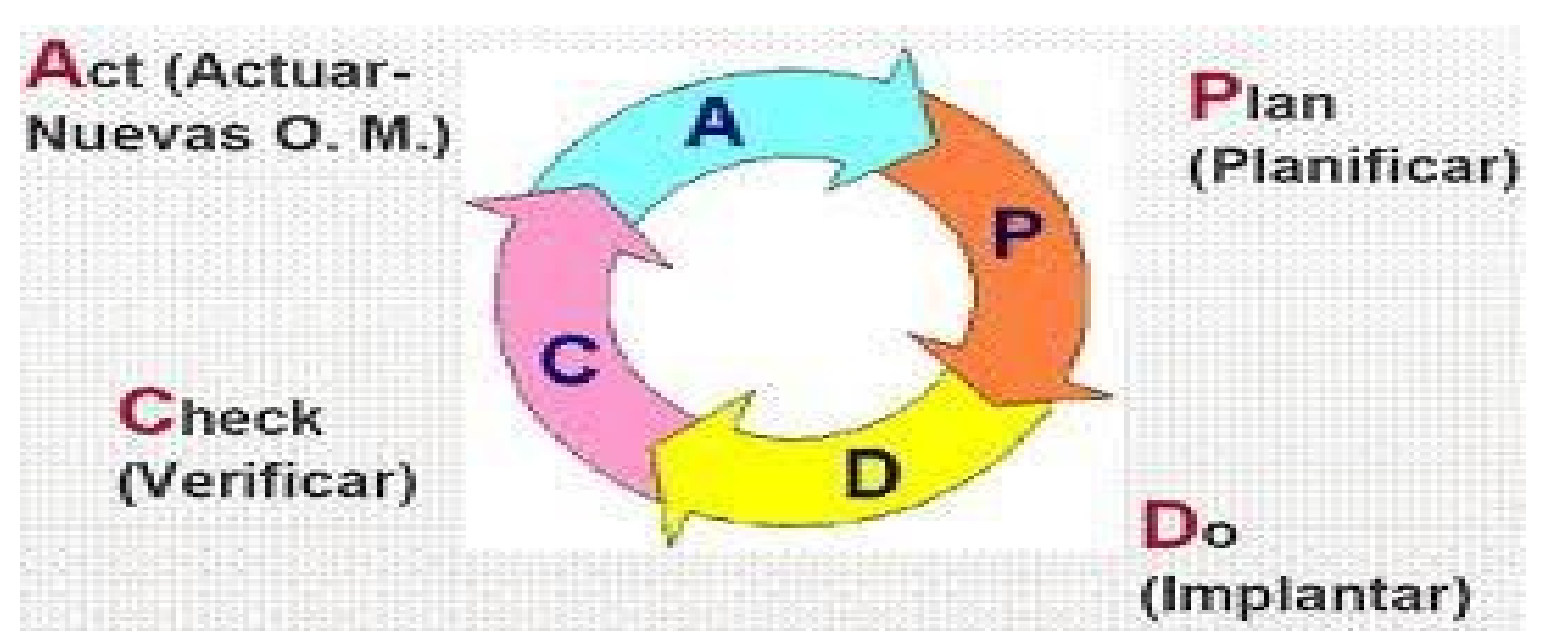

Figura 1. Ciclo básico de retroalimentación

Fuente: E. Deming (1997)

\section{i. Etapa de planificación}

En la etapa de planificación se va a estructurar el marco en el que se desarrollará la acción del VC, una vez definido será necesario plasmar por escrito los objetivos: ¿por qué hacemos un programa de VC?; los resultados previstos: ¿qué esperamos obtener de la participación o incorporación del VC en nuestra organización?; las actividades previstas: ¿qué es lo que realizaremos para conseguirlo?; y los recursos necesarios ¿qué recursos vamos a utilizar?. En esta etapa es aconsejable utilizar técnicas de braimstorming y para guiarlo es conveniente plantear un análisis de riesgos y beneficios de la colaboración con la empresa como plantean algunos autores (VALOR, 2010; LEMONCHE, 2011, ALLEN, 2012). En esta etapa se procede a la estructuración del marco en el que se desarrollará la acción del VC, tiene como punto de partida la estrategia global de la empresa, la misión y la visión así como los valores organizativos y ha de establecerse la forma de realizar la búsqueda de Organizaciones No Lucrativas susceptibles de establecer una colaboración de VC (FUNDACIÓN LA CAIXA, 2009).

También es necesario explicitar los potenciales beneficios para la empresa a nivel organizacional y de reputación corporativa. El VC contribuye a mejorar el clima organizacional a través del aumento de la motivación, satisfacción y sentimiento de pertenencia de los empleados (HARRIS, 2000). Asimismo, incide positivamente en la imagen corporativa y en el posicionamiento en el ámbito de la RSC, además posibilita la integración de los empleados en la acción social de la empresa (HOUGHTON et al. 2009; FUNDACIÓN CODESPA, 2012).

De acuerdo con Lemonche (2011) a pesar de que pueden existir múltiples fórmulas a través de las cuales los empleados de una empresa pueden organizar su participación en programas de VC se pueden plantear diferentes opciones atendiendo a la capacitación necesaria del voluntariado, al destino donde se desarrolle la tarea de voluntariado, a las causas que se persigan, a los campos de actuación, a las funciones a realizar y al tiempo empleado en el voluntariado. Hay que tener en cuenta que una vez comienza la incorporación del empleado al VC se inicia un proceso de intercambio en el cual el voluntario entrega a la ONL su ilusión, motivación y compromiso. Y por su parte, la ONL le proporciona al voluntario unos objetivos por los que trabajar, un equipo en el que integrarse, unos instrumentos de trabajo y un reconocimiento que ha de generarle satisfacción. 


\section{ii. La etapa de implementación}

En esta etapa es importante considerar el modelo de VC que va a seguir la empresa en línea con su estrategia de RSC. Ya que se ha de programar, gestionar y coordinar las actividades previstas a desempeñar por los voluntarios estableciendo la cronología de desarrollo y las normas de procedimiento necesarias. En este sentido, Lemonche (2011) identifica múltiples modelos de VC que exigen distinto compromiso por parte de la empresa:

- Donaciones, es la forma más sencilla y directa de participación solidaria de los empleados de una empresa en los programas de acción social de ésta mediante aportaciones voluntarias de dinero;

- Matching individual, la empresa organiza unidades de trabajos específicos para canalizar iniciativas de voluntariado de sus empleados;

- Microvoluntariado virtual, que consiste en que los empleados realicen pequeñas tareas a distancia sin desplazarse del puesto de trabajo;

- Microdonaciones de empleados, los empleados contribuyen con aportaciones únicas o periódicas de una cantidad de dinero muy pequeña;

- Campañas, se trata de un VC clásico en el entorno empresarial español, los empleados participan en actos mediante donaciones en dinero o en especie, o colaborando directamente en actividades que no requieren una especial cualificación técnica;

- Bancos de tiempo, los empleados utilizan una cuota de tiempo que la empresa concede para este fin y éstos aportan a su actividad voluntaria un tiempo equivalente de sus horas libres como complemente a la cuota de tiempo aportada por la empresa;

- Outdoor solidario, para los empleados supone una importante experiencia vital y tiene una gran capacidad de motivación y generación de espíritu de equipo entre los empleados;

- Voluntariado profesional, la persona voluntaria aplica sus conocimientos y habilidades profesionales en servicio de la ONL, en este caso existe una gran alineación en las competencias de la empresa y de la ONL;

- Cesión de capital humano, la empresa cede personal cualificado a la ONL durante un periodo de tiempo determinado, se trata de que el empleado trabaje temporalmente y de forma continua en la ONL, manteniendo la relación laboral con la empresa.

El desarrollo de la acción voluntaria supone gestionar adecuadamente la distribución de tareas y responsabilidades, de forma que la participación del voluntariado sea real en la ONL. Para ello, tanto la empresa como la ONL en la que se desarrolla el programa de voluntariado deben colaborar en el seguimiento y acompañamiento de la acción voluntaria.

\section{iii. Etapas de evaluación y actuación}

La etapa de evaluación tiene como objetivo conocer la evolución del programa de VC, en ella se realiza el seguimiento de la acción voluntaria y el análisis de la ejecución del programa identificando las causas de posibles de desviaciones o deficiencias detectadas (LÓPEZSALAS, 2009), y a partir de las mismas proponer medidas reconductoras del programa. En este punto, resulta de gran importancia la labor del responsable del VC y la realización de evaluaciones periódicas de la tarea llevada a cabo por los voluntarios. La evaluación constituye una oportunidad de dar y recibir retroalimentación y de analizar las metas logradas hasta el momento.

Por último, la etapa de actuación en busca de nuevas oportunidades consiste en, una vez identificadas las causas de posibles desviaciones o deficiencias, elaborar y proponer medidas reconductoras o alternativas que mejoren el VC. Se trata de volver a definir las prioridades y objetivos de la relación de la empresa con la ONL para analizar la coherencia del proyecto específico desarrollado con la línea de actuación de la RSC. Es en este momento en el que hay que pararse a pensar en lo que se ha estado 
haciendo hasta el momento y considerar si en realidad se ha aprovechado de forma correcta todo lo que pueden ofrecer las iniciativas de VC que se han llevado a cabo.

\section{Los recursos humanos y el voluntariado corporativo}

Según Ces y Adecco (2011, p. 22), a la hora de implantar un programa de voluntariado en una empresa, es importante seguir el mismo proceso que con cualquier otra iniciativa corporativa que implique la movilización y gestión de recursos y de capacidades dentro de la organización. Es decir, en primer lugar, cabe promover una reflexión dentro de la empresa sobre el alcance pretendido con estas actividades y el grado de alineamiento de éstas con los valores y la cultura de la organización.

En este apartado se explora y reflexiona sobre los recursos humanos, en base a las políticas de VC como una línea fundamental dentro de las políticas de RSC de las empresas. Cuando hablamos de VC, hablamos, también, de la organización y estructuración de estas políticas y del desarrollo de las actividades que conlleva, ya que el VC tiene una complejidad elevada, debido a los diversos factores que involucra (diferentes objetivos de las empresas y las ONG, expectativas creadas en los empleados, diferente visión de la rentabilidad de estas actividades, importancia de la calidad de la ayuda a los beneficiarios, etc.).

Para Valor (2010), el VC implica en la puesta de activos empresariales al servicio de proyectos sociales. Esta inversión social puede describirse como un mix de recursos de todo tipo, que puede contener:

1. Activos financieros: Donaciones en efectivo, programas de patrocinio asociados a fines sociales, préstamos de capital.

2. Activos tangibles no financieros: Productos de catálogo, espacio en almacenes, espacio en oficinas, maquinaria, medios de distribución, espacio en internet, excedentes de producción.

3. Activos intangibles: Elementos de imagen y marca, copyright y patentes, tecnología, I+D, beneficios asociados a contratos de suministro, organización de eventos.

4. Capital humano: Tiempo y capacidades de los empleados para programas de voluntariado corporativo.

Por sus características, complejidades y papel jugado, es el capital humano la inversión más importante del compromiso solidario de una empresa hacia las necesidades de la sociedad, ya que tiene que ver con la aportación del activo más valioso de la empresa que es su capital humano.

Según Valor (2010), el enorme potencial de talento, capacidades y entusiasmo de una plantilla formada, organizada y con espíritu solidario encuentra una vía inmejorable de aportación a la sociedad a través del VC. Todo ello hace necesario el desarrollo de herramientas que sirvan a las corporaciones para tomar decisiones adecuadas en este ámbito; ya que está en juego el prestigio de la empresa, la satisfacción de los empleados y la ayuda eficaz a colectivos necesitados.

Las recientes investigaciones muestran que desde el año de 2011 ha crecido a nivel mundial el número de empresas que incluyen el VC entre sus políticas de recursos humanos y de responsabilidad social. Sin embargo, dicha evolución presenta un panorama un tanto estancado en cuanto a la gestión del voluntariado (VALOR, 2010).

Para el Observatorio de Voluntariado Corporativo, esta situación puede ser síntoma de la necesidad de dar un nuevo paso hacia un VC verdaderamente estratégico, inseparable del propio negocio y de la cultura de la empresa. Según el OVC, este es un gran reto para quienes son responsables de su gestión en las compañías y también en las organizaciones del tercer sector. Entre las tareas pendientes para lograr la madurez del VC son señaladas las siguientes:

1. Conocer mejor las motivaciones de los empleados para fortalecer su implicación;

2. Mejorar la eficacia y la eficiencia de las iniciativas, a través del uso de métricas adecuadas que permitan conocer todas las dimensiones del impacto de un programa de voluntariado; 
3. Definir clara y explícitamente los objetivos de la estrategia y los diferentes planes de VC;

4. Innovar la oferta de acciones posibles, explorando nuevas herramientas, posibilidades de la cooperación sectorial y ampliando el espectro de grupos humanos con necesidades específicas.

En definitiva, el objetivo es consolidar un estilo de gestión del voluntariado que busque la excelencia a través de la gestión innovadora y eficiente de sus recursos humanos, es decir, las personas que integran la organización.

Sin embargo, estudios sobre el VC, como la investigaciones desarrollada por Voluntare (2013), señalan la importancia y necesidad de medir el impacto que se está teniendo sobre los diferentes actores involucrados a través del desarrollo de la acción voluntaria. La literatura indica que la medición del impacto del VC sigue siendo un tema pendiente entre las empresas y, en general, tampoco se evalúa la actuación y el resultado aportados por aquellos que colaboran en este marco de VC.

El hecho de no se medir el resultado del VC no permite mejorar su contribución, ni gestionarlo, y ni siquiera argumentar beneficios tangibles para garantizar una sostenibilidad en el compromiso de los recursos humanos y de la propia empresa. Para Voluntare (2013, p. 138), "las razones más comunes por la que la medición presenta un reto para empresas y sus recursos humanos en el ámbito de VC son varias y se podrían definir en tres aspectos. En primer lugar, la medición de los resultados e impactos de los programas de voluntariado corporativo puede suponer un reto debido a la orientación de la mayoría de las empresas hacía la acción. Es decir, el enfoque se centra en el desarrollo e implantación de actividades de voluntariado dificultando el establecimiento de hitos de medición. En segundo lugar, la medición de los impactos de este tipo de programas se encuentra con escepticismo interno. Los ejercicio de medición requieren una asignación de recursos por parte de la empresa - sea humanos o financieros.

Debido a esta orientación a la acción, la asignación de recursos para el desarrollo de la medición puede parecer, internamente, como un aspecto eliminable ya que se considera preferible el desarrollo de actividades. Y finalmente, existen casos donde la empresa o los responsables del programa pueden tener su propio escepticismo o fobia a la medición del impacto de este tipo de programas ya que destaca una intención de la empresa por la mejora del programa - destacando puntos débiles - y una mayor integración estratégica en la gestión de la empresa. Este tipo de ejercicios de medición, en definitiva, llevan a la mejor gestión y comprensión del programa planteando diferentes opciones de avance que pueden ser vistas positivamente o recibidas con el escepticismo interno comentado anteriormente."

Más allá de una orientación hacía la acción y el escepticismo interno, el ejercicio de medición de impacto, también está condicionado por los objetivos que persiguen los colaboradores. Una empresa, por ejemplo, puede tener diferentes objetivos a la hora de desarrollar acciones en el marco del VC. Desde los recursos humanos, el VC es un instrumento con un alto nivel de coste-efectividad para el desarrollo de habilidades y capacidades. Permite, entre otras cosas, aprender y practicar habilidades, desarrollar capacidades de liderazgo, aprender a trabajar eficazmente en equipo, refinar las habilidades existentes, trabajar en nuevos entornos y construir nuevos conocimientos de las realidades sociales. Desde un punto de vista más corporativo, puede querer mejorar su reputación e imagen entre sus grupos de interés locales o posicionarse como un colaborador activo y fiable en el apoyo social de su comunidad (VOLUNTARE, 2013, p. 139).

Por lo tanto, la medición de impacto de programas de VC sigue siendo un reto para empresas, entidades y otros colaboradores que puedan estar involucrados en el desarrollo de acciones de voluntariado. Algunas empresas con prácticas más avanzadas de voluntariado suelen incluir indicadores de medición tales como el nivel de satisfacción de los empleados y el impacto social cuantitativo (número de beneficiarios, horas dedicadas, etc.). Por esa razón, muchas empresas, ante el riesgo de que no haya una gran participación de empleados, tienden a ofrecer acciones que consideran pueden ser divertidas para la plantilla. Esto en muchos casos supone un coste de oportunidad frente al potencial impacto social (VOLUNTARE, 2013, p. 15).

Actualmente, ya existe disponible para las empresas un gran número de herramientas y metodologías de medición del impacto que se está teniendo sobre los diferentes actores involucrados a través del desarrollo de la acción voluntaria. En el ámbito de los recursos humanos, la medición de resultados de programas de $\mathrm{VC}$ debe incorporar herramientas y metodologías que intentan brindar apoyo 
al ejercicio de medición de los resultados de programas de voluntariado corporativo, de modo a motivar la persuasión interna en la propia empresa. Eso porque las empresas no están aprovechando bien sus programas de VC para medir y entender los beneficios asociados con el desarrollo de capacidades y habilidades de sus recursos humanos.

Además de facilitar la labor de destacar la importancia de las acciones llevadas a cabo por los empleados, el hecho de incorporar la medición del resultado de los recursos humanos como parte integrante de la gestión de los programas de VC, al igual que se hace en cualquier otro proyecto empresarial, tiene impacto positivo en la contribución que tiene el VC para la empresa y para la sociedad. De ahí que una metodología desarrollada para medir el impacto que se está teniendo sobre los diferentes actores involucrados a través del desarrollo de la acción voluntaria debe:

1. Adoptar un enfoque estratégico, identificando a los objetivos clave que están tratando de lograr.

2. Adoptar una aproximación estratégica de los recursos humanos.

3. Construir un marco referente a las actividades, recursos y actores del programa. empresa.

4. Identificar las métricas existentes, se posible aprovechando las medidas utilizadas por la

5. Analizar a las diferencias métricas y modificar los gaps según sea necesario.

6. Dialogar con grupos de interés, involucrando al personal que tiene la responsabilidad de gestión.

Lemonche (2011, p. 93) señala que la medición de resultados es una necesidad y un reto aplicable a las actividades voluntarias, para que se pueda gestionar al VC. Por ser el VC una herramienta de generación de valor, es necesario evaluar esta aportación de valor para los agentes que participan en ella, estableciendo indicadores, criterios de seguimiento y mecanismos de medición y valoración de resultados.

\section{Conclusiones}

El VC es una realidad que está creciendo y que previsiblemente va a adquirir una mayor presencia en el futuro. Hay que señalar que el VC como estrategia empresarial sirve para afianzar las políticas de recursos humanos en la empresa y para redefinir la RSC contribuyendo a una mejora de su entorno social. La implementación del VC por parte de la empresa debe permitir perseguir el equilibrio entre la responsabilidad interna y externa procurando atender las necesidades y demandas de los diferentes grupos de interés. Y es que el VC favorece la mejora del clima organizacional y la imagen corporativa de la empresa.

El VC representa una oportunidad para motivar a los empleados a través de la participación en el programa de VC, para lo cual se requiere profundizar en su gestión. La gestión del programa de VC es compleja, por lo que requiere un responsable de $\mathrm{VC}$, ya que se ha de gestionar la participación de los empleados en el programa y la relación con la ONL en la que se va a desarrollar el programa para que la colaboración sea duradera, a largo plazo y se adapte a los intereses de la empresa.

En este trabajo hemos prestado atención a la gestión del VC desde una perspectiva teórico-analítica con el objetivo de contribuir al debate sobre esta incipiente y creciente práctica de voluntariado. Dada la importancia de profundizar en el proceso de implementación de programas de VC, se ha puesto de relieve que éste se tiene que conducir de forma similar al proceso que sigue cualquier organización al establecer su control de gestión según el ciclo básico de retroalimentación con sus diferentes etapas: planificación, implementación, evaluación y actuación.

La etapa de planificación debe responder a preguntas como las siguientes: ¿Qué es el VC y cuál es la situación del VC? ¿Qué pretendemos y qué esperamos obtener a partir de la participación en el VC? También hay que determinar la coherencia del VC con la estrategia de RSC de la empresa, su perfil y políticas. En ella se estructura la acción quedando establecidos y definidos todos los elementos del 
programa de VC. La etapa de implementación contempla el desarrollo de la acción voluntaria y el acompañamiento del empleado en el programa de voluntariado en la ONL. La etapa de evaluación tiene como finalidad el seguimiento y análisis de la acción voluntaria. Por último, la etapa de actuación trata de volver a definir las prioridades y objetivos de la relación de la empresa y la ONL para analizar la coherencia del proyecto específico desarrollado con la línea de actuación de la RSC.

A modo de conclusión, señalamos que para medir un programa de VC de manera plena, es importante tener en cuenta la gestión de los recursos humanos antes de empezar el programa - durante su conceptualización - y utilizar el ejercicio de medición de su resultado y desempeño como guía para mejorar la calidad del programa desde el punto de vista de valor para el empleado.

\section{BIBLIOGRAFÍA}

Aldefer, C. (1969). An empirical test of a new theory of human needs. Organizational Behavior and Human Performance, 142-175, May.

Allen, K. (2003). The social case for corporate volunteering. Australian Journal on Volunteering, 8, 57-62.

Allen, K. (2012). La gran Carpa Voluntariado corporativo en la era global. Madrid: Fundación telefónica/Ariel.

Alves, I.F. (2010). O papel do voluntariado empresarial como influenciador na gestão organizacional: um estudo de caso do Sistema Federação das Indústrias do Estado do Ceará. Fortaleza: FA\&7.

Azevedo, D. (2007). Voluntariado corporativo - motivações para o trabalho voluntário. (Artigo selecionado dos anais XXVII Encontro Nacional de Engenharia de Produção). Revista Produção On Line, Edição especial/dezembro.

Canto, A.; Cabezas, I.; Vidorreta, I. (2010). Las organizaciones de voluntariado en la CAPV: realidad actual y retos. Zerbizuan, 48, 109-116.

Ces \& Adecco. (2011). Estudio sobre el estado del voluntariado corporativo en España 2010. El valor del voluntariado corporativo para la empresa y sus colaboradores. Madrid: Fundación Adecco y Club de Excelencia en Sostenibilidad.

Chacon, F.; Pérez, T.; Flores, J.; Vecina, M.L. (2010). Motivos del voluntariado: Categorización de las motivaciones de los voluntarios mediante pregunta abierta. Intervención Psicosocial, 19(3), 213-222.

Cnaan, R.A.; Goldberg-Glen, R.S. (1991). Measuring motivations to volunteer in human services. Journal of Applied Behavioral Science, 27, 269-284.

Comunidade Solidária. (1997). Proposta de Criação de Centros de Voluntários. Relatório do processo de consulta. Brasília.

Corullón, M. (2014). Trabalho Voluntário: Manual elaborado para o Programa de Promoção do Voluntariado do Conselho Comunidade Solidaria. Disponible en: http://www.santafedosul.sp.gov.br/sfsspgovbr/voluntario/pg805.htm (consultado en 13 abril 2014)

Costa, E.P.M. (2004). O sentido do trabalho voluntário e o terceiro setor. Dissertação de mestrado, Universidade de Taubaté. Taubaté, SP, Brasil.

Deming, E. (1997). La nueva economía: Para la industria, el gobierno y la educación. Madrid: Diaz de Santos.

Fresno, J.M.; Tsolakis, A. (2012). Profundizar en el voluntariado: Los retos hasta 2020. Madrid: Plataforma del Voluntariado de España (PVE) Disponible en: http://www.plataformavoluntariado.org/observatorio-publicaciones.php (consultado en 12/08/2015).

Fundación Codespa. (2012). Guía práctica para la implementación de iniciativas de Voluntariado Corporativo para el Desarrollo. En: Fundación Codespa, Voluntariado Corporativo para el desarrollo. Una herramienta estratégica para integrar empresa y empleados en la lucha contra la pobreza. p. 85-92.

Gallardo Vázquez, D.; Sanchez Hernández, M.I. (2014). Responsabilidad social en las universidades: propuesta de un programa de voluntariado corporativo. Trabajo presentado en las I Jornadas Internacionales sobre Responsabilidad Social Universitaria, realizada en Cádiz.

Gallardo Vázquez, D.; Sanchez Hernández, M.I.; Corchuelo Martínez, M.B.; Guerra Guerra, A. (2010). Diagnóstico del voluntariado corporativo en la empresa española. Revista de Estudios Empresariales, (2), 54-80.

Garay, A.B.B.S. (2001). Programa de voluntariado empresarial: modismo ou elemento estratégico para as organizações. Revista de Administração, 36(3), 6-14, jul/set. 
Garay, A.B.B.S.; Mazzilli, C.P. (2003). Uma análise do(s) significado(s) do trabalho voluntário empresarial. REAd Revista Eletrônica de Administração, 9(5), Ed. 35, 1-17, set/out., set/out.

Geber, B. (1991). Managing Volunteers. Training.Minneapolis, 28(6), 21-26, junho.

Harris, E. (2000). Corporate giving goes both ways: Companies that offer volunteerism are attracting top talent and motivating employees. Sales and Marketing Management, 152(12), 104.

Hersberg, F. (1966). Work and nature of man. Cleveland, Ohio: The World Publishing Co.

Houghton, S.M.; Gabel, J.T.A.; Williams, D.W. (2009). Connecting the two faces of CSR: Does employee volunteerism improve compliance? Journal of Business Ethics, 87, 477-494.

Jarver, C.; Parker, A. (2012). Programas de voluntariado corporativo: siete pasos para el éxito. En Fundación Codespa, Voluntariado Corporativo para el desarrollo. Una herramienta estratégica para integrar empresa y empleados en la lucha contra la pobreza. p. 95-105.

Kotler, P.; Lee, N. (2005). Corporate Social Responsibility: doing the most good for your company and your cause. New York: John Wiley and Sons Inc.

Latham, G.P.; Pinder, C.C. (2005). Work motivation theory and research at the dawn of the Twenty-First Century. Annual Reviews Psychology, 56, 485-516.

Lemonche, P. (2011). Voluntariado corporativo, un puente de colaboración entre la empresa y la sociedad. Madrid: Forética.

Lima, A.J.F.S.; Bareli, P. (2011). A importância social do desenvolvimento do trabalho voluntário. Disponible en: www.eticaempresarial.com.br/imagens.../File/.../artigo_voluntariado.pdf (consultado en 13 abril 2014)

Locke, E. (1968). Toward a theory of task motivation and incentives. Organizational Behavior and Human Performance, 157-189, May.

López Salas, E. (2009). Claves para la Gestión del Voluntariado en las Entidades no Lucrativas. Madrid: Fundación Luis Vives.

Loro, S.; Conde, C. (2001). Ideas para maximizar los beneficios del Voluntariado Corporativo para el Desarrollo. Madrid: Fundación Codespa.

Maslow, A.H. (1954). Motivation and personality. New York: Harper \& Row Publishers.

McClelland, D.C. (1965). Toward a theory of motive acquisition. American Psychologist, 20, 321-333.

Observatorio del Tercer Sector. (2009). Manual de gestión del voluntariado. Barcelona: Fundación "La Caixa".

Observatorio del Tercer Sector. (2007). Buenas prácticas en la gestión del voluntariado. Barcelona: Fundación "La Caixa".

Observatorio del Tercer Sector. (2013). Los retos del voluntariado corporativo en las empresas españolas. Disponible en: http://www.observatoriovc.org/observatorio-vc/ (consultado en: 14/11/2015).

Peloza, J.; Hassay, D.N. (2006). Intra-Organizational Volunteerism: Good Soldiers, Good Deeds and Good Politics. Journal of Business Ethics, 64, 357-379.

Pittman, T.; Heller, J.F. (1987). Social motivation. Annual Reviews Psychology, 38, 461-489.

Richardson, R.J. (1999). Pesquisa social: métodos e técnicas. (3a ed.). São Paulo: Atlas.

Rosenfeld, S.I.; Muterle, M.C.C.; Gaspar, J.A. (2013). Programa de voluntariado empresarial: reflexões sobre o caso da Transpetro em São Sebastião. Trabajo presentado en el ADM 2013 - Congreso Internacional de Administración, Ponta Grossa, PR, 23-27/09/2013.

Sanz, B.; Cordobés, M.; Calvet, A. (2012). El voluntariado corporativo en España. Modelos y perspectivas de impacto social. Madrid: Instituto de Innovación Social de Esade.

Silva, J.O. [org.] (2004) Novo voluntariado social: teoria e ação. Porto Alegre: Dacasa.

Silva, J.O.; Feitosa, S. (2002). Ação Social Voluntária: Motivação e Evasão. Cadernos Cedope, 13(19).

Simon, H.A. (1965). Comportamento administrativo. Rio de Janeiro: Fundação Getulio Vargas. 
Souza, S.A. (2008). Educação, trabalho voluntário e responsabilidade social da empresa: "amigos da escola" e outras formas de participação. Tesis doctoral, Universidade de São Paulo, São Paulo, SP, Brasil.

Teodósio, A.S.S. (2001). Programas de incentivo ao voluntariado: novos desafios para a ética gerencial. (Trabajo presentado en el IV Congreso Latinoamericano de Ética, Negocios y Economía). Buenos Aires: Alene/Uade, p. 17-27.

Tschiart, M. (2005). Employee volunteer programs. In: Brundnew, J. L. (ed.) Emerging areas of volunteering. Indianapolis: Arnova, p. 13-29.

Valor Martínez, C. [coord.] (2010). Relaciones con la sociedad. (Colección Sostenibilidad y Responsabilidad Social Corporativa) A Coruña: Editorial Netbiblo.

Vecina, M.L.; Chacón, F. (2009). Satisfacción en el voluntariado: estructura interna y relación con la permanencia en las organizaciones. Psicothema, 21(1), 112-117.

Veras, A.I.G. (2012). Voluntariado corporativo: a importância do voluntariado para o desenvolvimento de competência: um estudo de caso na Coelce. Fortaleza: FA7.

Voluntare. (2013). Guía de voluntariado corporativo. Madrid: Voluntare Red de Voluntariado Corporativo. Disponible en: www.voluntare.org (consultado en 13 abril 2014)

Vroom, V.H. (1964). Work and motivation. New York: John Wiley \& Sons. 